\title{
Evidence of Functional Mossy Fiber Sprouting in Hippocampal Formation of Kainic Acid-Treated Rats
}

\author{
DAVID L. TAUCK ${ }^{* 2}$ AND J. VICTOR NADLER ${ }^{\ddagger 3}$ \\ Departments of * Physiology and $\ddagger$ Pharmacology, Duke University Medical Center, Durham, North Carolina 27710
}

\begin{abstract}
In the rat hippocampal formation, degeneration of CA4derived afferent fibers provokes the growth of mossy fiber collaterals into the fascia dentata. These aberrant fibers subsequently form granule cell-granule cell synapses. The hippocampal slice preparation was employed to determine whether these recurrent connections are electrophysiologically functional. Hippocampal slices were prepared 12 to 21 days after the bilateral destruction of CA4 neurons with either intracerebroventricular or intravenous kainic acid (KA). In slices from control rats, antidromic stimulation of the mossy fibers elicited a single population spike in the granular layer of the fascia dentata. In contrast, when slices from some KAtreated rats were similarly tested, antidromic stimulation elicited multiple population spikes. This effect was not reproduced by blocking inhibitory transmission with bicuculline methiodide. Slices from other KA-treated rats fired a single population spike, but an antidromic conditioning volley increased the amplitude of a subsequent antidromic population spike by 5 to $15 \%$. In slices from control rats, on the other hand, an antidromic conditioning volley always either decreased or failed to alter the amplitude of an antidromic test response. Superfusion with $\mathrm{Ca}^{2+}$-free medium containing 3.8 $\mathrm{mm} \mathrm{Mg}^{2+}$ reverslbly abolished all effects of $\mathrm{KA}$ administration. Abnormal responses to antidromic stimulation correlated with the loss of CA4 neurons and the growth of supragranular mossy fiber collaterals in the same animals.

These results suggest that supragranular mossy fiber collateral sprouts form a functional recurrent excitatory circuit. These aberrant connections may further compromise hippocampal function already disrupted by neuronal degeneration, such as by facilitating seizure activity.
\end{abstract}

Administration of the potent convulsant kainic acid (KA) reproduces in rats lesions remarkably similar to Ammon's horn sclerosis (AHS) in humans (for review, see Nadler, 1981). AHS, a well recognized pathological finding in the brains of temporal lobe epileptics,

Received October 20, 1983; Revised August 23, 1984;

Accepted October 3, 1984

' We thank Dr. M. Gruenthal for assistance with the statistical analyses and Drs. W. A. Wilson, G. Somjen, and B. Connors for helpful advice and discussions. This study was supported by National Institutes of Health Grants NS 06233 and NS 17771 and Research Career Development Award NS 00447

${ }^{2}$ Present address: Department of Anesthesia, Stanford University School of Medicine, Stanford, CA 94305.

${ }^{3}$ To whom correspondence should be addressed, at Department of Pharmacology, Duke University Medical Center, Durham, NC 27710. involves extensive degeneration of hippocampal pyramidal cells, with sparing of granule cells (Corsellis and Meldrum, 1976). The effect of clinical or experimentally induced AHS on hippocampusdependent behaviors and on epileptogenesis depends not only on the loss of hippocampal neurons and their connections, but also on any synaptic rearrangements that result from this lesion. Such changes in connectivity have been demonstrated after a variety of lesions to the hippocampal area of experimental animals (reviewed by Cotman and Nadler, 1978), and they are likely to occur also in the brains of temporal lobe epileptics (De Lorenzo and Glaser, 1981; De Lorenzo et al., 1982).

Clinical and KA-induced experimental AHS involve the degeneration of hippocampal CA4 neurons (that is, the morphologically diverse neurons that lie in the dentate hilus between the dendrites of area CA3C pyramidal cells and the hilar border of the granular layer) as well as pyramidal cells. CA4 neurons give rise to the associational and commissural pathways that terminate on the proximal third of granule cell dendrites in the ipsilateral and contralateral fascia dentata, respectively (Gottlieb and Cowan, 1973; Swanson et al., 1978; Berger et al., 1981; Laurberg and Sørensen, 1981). Degenerating associational-commissural afferent fibers are replaced, in part, by nascent collaterals of the hippocampal mossy fibers (axons of the dentate granule cells) (Nadler et al., 1980). Mossy fiber collateral sprouts grow across the granular layer of the fascia dentata and form a plexus in the denervated lamina. Such mossy fiber growth can be provoked not only by KA lesions, but also by other lesions of the associational-commissural projection (Laurberg and Zimmer, 1981). At least some of these aberrant fibers make synaptic connections with dendrites of granule cells (Frotscher and Zimmer, 1983). Thus, destruction of CA4-derived projections creates aberrant granule cell-granule cell connections in the rat fascia dentata.

If these recurrent mossy fiber synapses were electrophysiologically functional, they would endow the granule cells with excitatory input that is not normally present. A single stimulus might then elicit repetitive discharge. We have explored this possibility with use of the hippocampal slice preparation. Preliminary reports of this work have been presented (Tauck and Nadler, 1982; Nadler et al., 1983).

\section{Materials and Methods}

$K A$ lesions. Male Sprague-Dawley rats ( 45 to 60 days of age; Harlan Sprague-Dawley, Madison, WI) were injected with KA either intracerebroventricularly (ICV) or intravenously. For ICV administration, rats were anesthetized with pentobarbital (55 mg/kg, i.p.), and 2.34 to $3.75 \mathrm{nmol}$ of $\mathrm{KA}$ in a 1 $\mu$ l volume of artificial cerebrospinal fluid (Elliott, 1969) were injected during a 30-min period into each lateral ventricle. For intravenous administration, unanesthetized rats were injected in a lateral tail vein with $11 \mathrm{mg} / \mathrm{kg}$ of $\mathrm{KA}$ dissolved in isotonic saline. Intravenous injections usually destroyed a greater percentage of CA4 neurons that did ICV injections, and they more consistently provoked the growth of supragranular mossy fiber collaterals. Otherwise, results did not differ with the route of administration. Control animals were injected with the appropriate vehicle alone or were not injected. Data from both types of control were indistinguishable.

Preparation of hippocampal slices. Hippocampal slices were prepared 12 
to 21 days after administration of KA or vehicle. This interval was chosen because the growth of mossy fiber collaterals is already maximal in 12 days (Laurberg and Zimmer, 1981). We observed no obvious difference in electro physiological or morphological data between slices from rats that survived 12 or 21 days after KA administration.

Alternate transverse slices of $500 \mu \mathrm{m}$ thickness cut from the middle third of the hippocampal formation were used for electrophysiological studies. The remaining slices from the same hippocampi were saved for histological analysis. For electrophysiological studies, hippocampal slices were suspended upon a nylon net in recording chambers of the type described by White et al. (1978). Elliott's (1969) artificial cerebrospinal fluid (composition: $122 \mathrm{~mm} \mathrm{NaCl}, 25 \mathrm{~mm} \mathrm{NaHCO}_{3}, 3.1 \mathrm{~mm} \mathrm{KCl}, 1.3 \mathrm{~mm} \mathrm{CaCl}_{2}, 1.2 \mathrm{~mm} \mathrm{MgSO}_{4}$, $0.4 \mathrm{mM} \mathrm{KH}_{2} \mathrm{PO}_{4}$, and $10 \mathrm{mM}$ D-glucose ( $\left.\mathrm{pH} 7.4\right)$, gassed continuously with water-saturated $95 \% \mathrm{O}_{2}: 5 \% \mathrm{CO}_{2}$ ) was recirculated through each superfusion chamber for a 1.5- to 2-hr period before experimentation commenced. During this time the fluid level was maintained just below the top surface of the slice. The temperature of the superfusion medium was $31 \pm 1^{\circ} \mathrm{C}$. Slices from KA-treated and control rats were studied either separately or concurrently; differences between the two groups were the same in either case. Blind electrophysiological analyses were not attempted, since cell death and gliosis were visually obvious in hippocampal slices from KA-treated rats.

Stimulation and recording. Cathodal constant current pulses of 50 - to $100-\mu \mathrm{sec}$ duration were applied through the inner wire of a concentric bipolar electrode (inner Pt/lr wire, $25 \mu \mathrm{m}$ in diameter; outer stainless steel cylinder, $100 \mu \mathrm{m}$ in diameter). Glass micropipettes filled with either $3 \mathrm{M} \mathrm{NaCl}$ or $2 \mathrm{M}$ $\mathrm{NaCl}, 1 \mathrm{M}$ sodium citrate ( 2 to 15 megohms impedance) were used to record extracellular field potentials. These signals were filtered above $1 \mathrm{kHz}$, amplified, displayed on an oscilloscope, and recorded on magnetic tape. Signals recorded on tape were digitized, averaged, and drawn with an X-Y plotter.

Antidromic population spikes were recorded from the granular layer of the suprapyramidal blade midway between the end and apex of the granule cell arch. The stimulating electrode was placed in the mossy fiber bundle where it exits the hilus. In slices from control rats, antidromic spikes were insensitive to superfusion with $\mathrm{Ca}^{2+}$-free medium and were abolished by selectively cutting the mossy fibers. Therefore, our antidromic volleys probably lacked any significant orthodromic component and did not fire the granule cells by spread of current to the cell bodies.

Mossy fibers were stimulated with paired pulses delivered at an interpair interval of $30 \mathrm{sec}$ or more. The amplitude (in millivolts) of antidromic granule cell population spikes displayed on the oscilloscope was measured from base line to peak negativity. Because the stimulus artifact sometimes obscured the onset of the antidromic spike, the base line was taken as the point just before onset of the second stimulus of the pair. Slices were retained for study only if maximal stimuli evoked at least an 8-mV antidromic population spike. The stimulus intensity was adjusted to evoke an initial submaximal antidromic population spike of 3 to $5 \mathrm{mV}$. Then stimulus pairs were delivered with various interstimulus intervals (ISIs). The mossy fibers were stimulated 5 to 10 times with each ISI, and amplitudes of the population spike(s) were averaged. The mean amplitude of the response to the second stimulus in each pair was compared to the mean amplitude of the response evoked by the first stimulus. In slices from some KA-treated rats, additional (presumably orthodromic) population spikes followed the antidromic spike. To measure the amplitude of these additional spikes, we determined the difference between apparent onset of the spike and its peak negativity and between its apparent offset and peak negativity. Spike amplitude was calculated by averaging these two values. Population spike amplitude serves as a measure of the number of granule cells brought to threshold by the stimulus, provided that the amplitude of individual action potentials remains constant (Andersen et al. 1971)

Application of experimental media. These studies were conducted with slices submerged in the superfusion medium. Media equilibrated with $95 \%$ $\mathrm{O}_{2}: 5 \% \mathrm{CO}_{2}$ were superfused at a rate of $2 \mathrm{ml} / \mathrm{min}$ from pressurized flasks. $\mathrm{Ca}^{2+}$-free medium was prepared by replacing $\mathrm{CaCl}_{2}$ with twice the concentration of $\mathrm{MgCl}_{2}$ (final $\mathrm{Mg}^{2+}$ concentration of $3.8 \mathrm{~mm}$ ). Electrophysiological responses were first obtained during superfusion with normal medium. Slices were then superfused with bicuculline methiodide (BMI)-containing or $\mathrm{Ca}^{2+}$. free medium for 10 to 15 min before any additional data were collected. By this time, stimulation of perforant path fibers in $\mathrm{Ca}^{2+}$-free medium no longer evoked a field potential, and in BMl-containing medium it evoked multiple population spikes (Ault and Nadler, 1983).

Histology. Alternate slices from the middle third of the hippocampal formation were reserved for heavy metal staining. These slices were maintained in buffered $\mathrm{Na}_{2} \mathrm{~S}$ solution (Danscher, 1981) for about $1 \mathrm{hr}$ and were then fixed in $10 \%$ neutral buffered formalin for about $2 \mathrm{hr}$. Each slice was then embedded flat in Tissue Tek II O.C.T. compound (Miles Laboratories, Naperville, IL) and cut into frozen sections. Sections were thaw-mounted onto coated sides and stained for the presence of heavy metal cations by Timm's sulfide silver method as modified by Danscher (1981). Additional slices were transferred to neutral buffered formalin directly after cutting, maintained in this solution for $3 \mathrm{hr}$, and then embedded and cut into frozen sections. Slide-mounted sections were stained with cresyl violet.

After all experiments had been completed, the histological sections were evaluated without knowledge of the electrophysiological data. Sections stained with cresyl violet were used to estimate the extent to which CA4 neurons had been destroyed. Those cut through the temporal third of the hippocampal formation were chosen for analysis, because the cell bodies
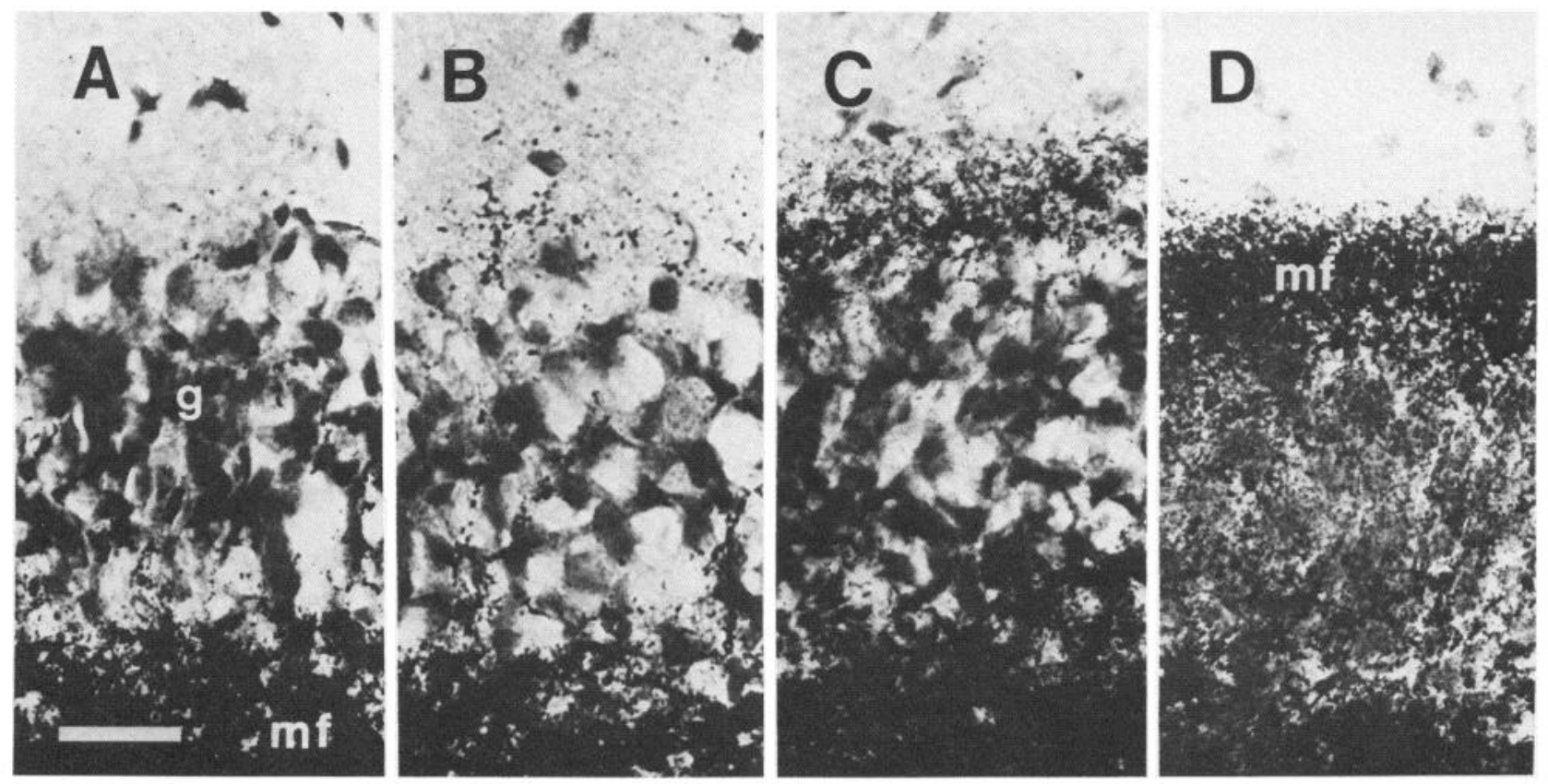

Figure 1. Effect of KA administration on the pattern of Timm's sulfide silver staining in the rat fascia dentata. Sections shown were counterstained with cresyl violet. $A$, Section from control rat. Timm's score of $0 . g$, granular layer; $m f$, mossy fibers. $B$ to $D$, Sections from rats treated with KA. $B$, Timm's score of 1 ; $C$, Timm's score of $2 ; D$, Timm's score of 3 . Note the dense supragranular band of mossy fiber-like staining in $D$. Calibration: 0.03 mm. 


\section{Control}

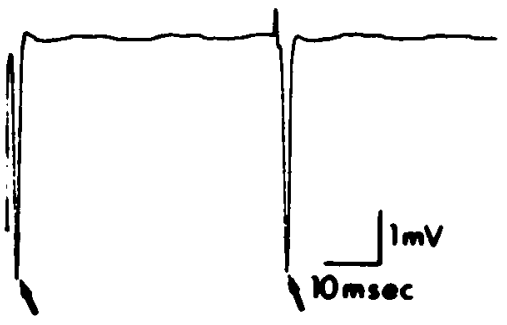

KA - Treated

Potentiation

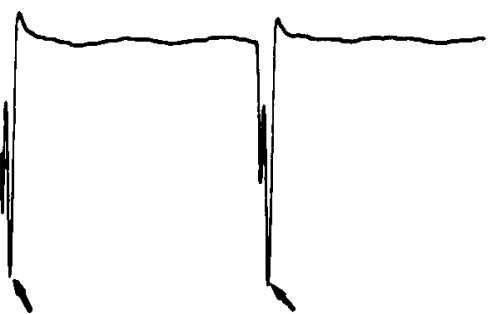

KA-Treated Multiple Firing

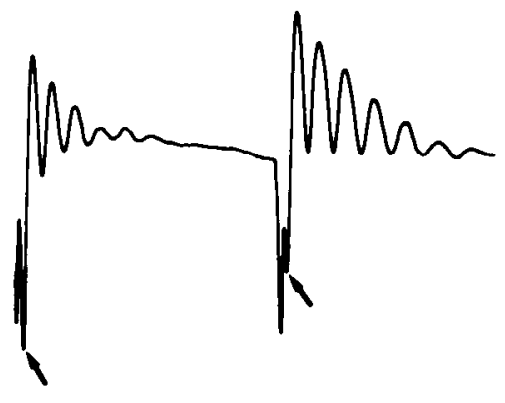

Figure 2. Effects of KA administration on the response of dentate granule cells to antidromic stimulation of the mossy fibers. A pair of identical submaximal electrical pulses were delivered with an ISI of $50 \mathrm{msec}$. Arrows here and in Figure 3 indicate the antidromic population spike. The X-Y plotter broadened the stimulus artifact associated with the second pulse of the pair.

from which associational-commissural fibers originate are mainly located there (Swanson et al. 1978). CA4 cells were counted in three sections from each animal with the aid of a camera lucida. Only cell fragments larger than $10 \mu \mathrm{m}$ in diameter were included in the count, thus excluding glia and displaced granule cells (Gaarskjaer and Laurberg, 1983). Values from each section were averaged.

Sections stained for the presence of heavy metal cations were used to estimate the extent of recurrent mossy fiber growth. Mossy fiber boutons were recognized by their characteristically black staining, indicative of their unusually high content of heavy metal cation, mainly $\mathrm{Zn}^{2+}$ (Haug, 1973). Sections from each animal were assigned a score from 0 to 3 related to the quantity of supragranular mossy fiber-like staining (Fig. 1). Sections with no or only occasional supragranular mossy fiber-like staining were scored 0 . Sections with scattered mossy fiber-like staining above all parts of the granular layer were given a score of 1 . Sections that exhibited either patches of heavy mossy fiber-like staining interspersed with regions of sparser staining or a continuous band of staining intermediate in intensity between sections scored 1 and 3 were given a score of 2 . Finally, sections with a dense, continuous band of supragranular mossy fiber-like staining were assigned a score of 3 .

\section{Results}

\section{Electrophysiology}

Slices from control rats. Antidromic activation of the granule cells elicited a single negative-going population spike in the granular layer (Fig. 2). When paired antidromic stimuli were delivered with ISIs of 15 to $120 \mathrm{msec}$, the response to the second stimulus of the pair was always smaller than or equal to that evoked by the first. Pairedpulse depression was greatest (about $10 \%$ ) with an ISI of less than $30 \mathrm{msec}$. Superfusion with neither $\mathrm{Ca}^{2+}$-free medium nor $100 \mu \mathrm{M}$ BMl significantly altered these results (Tauck, 1983). Accordingly, we conclude that paired-pulse depression of the antidromic population spike probably did not depend on recurrent GABAergic inhibition. As expected, however, $100 \mu \mathrm{M}$ BMl markedly depressed recurrent inhibition as assayed with antidromic-orthodromic stimulus pairs.

Slices from KA-treated rats. Slices from animals treatcd with KA could be divided into three groups, based on their response to antidromic stimulation of the mossy fibers. Slices from about half of the KA-treated rats were electrophysiologically indistinguishable from those of controls. Slices from six other KA-treated rats fired multiple population spikes in response to a single antidromic stimulus (Figs. 2 and 3 ). These negative-going spikes were separated from each other by 4 to $6 \mathrm{msec}$ and were superimposed on a positive wave whose amplitude varied directly with the total number of spikes. Such a response was never observed in slices from control rats. Superfusion with $\mathrm{Ca}^{2+}$-free medium reversibly abolished the second and succeeding population spikes of the multispike complex, as

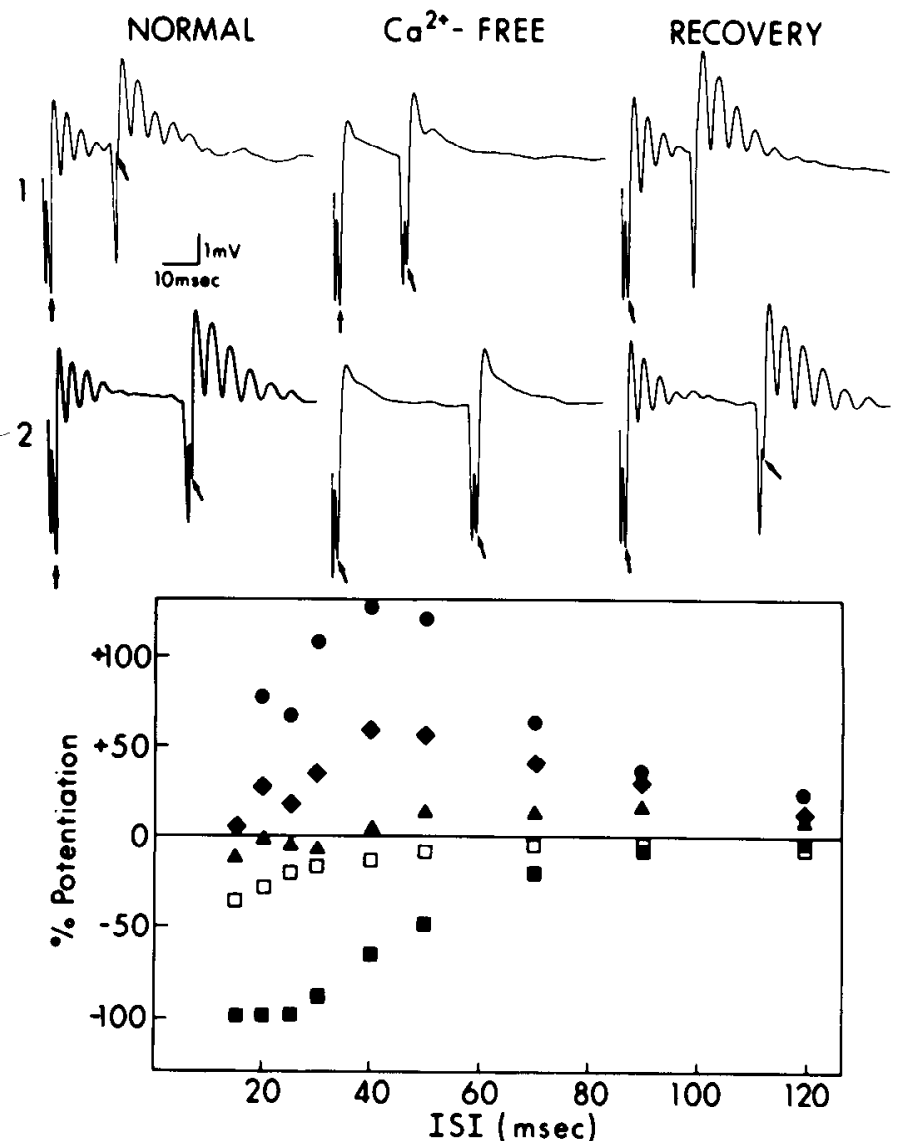

Figure 3. Multiple population spikes in response to paired submaximal antidromic stimuli in a slice from a KA-treated rat. Results are taken from an experiment in which the antidromic population spike was followed by several additional spikes. Top, 1:20 msec ISI; 2: $40 \mathrm{msec}$ ISI. Note that with an ISI of $20 \mathrm{msec}$ there was little evidence of an antidromic population spike in response to the second stimulus of the pair in normal medium. Bottom, Potentiation or depression of different components of the multispike complex by a conditioning volley as a function of ISI. $\mathbf{E}$, antidromic population spike; $\boldsymbol{\Lambda}$, first additional spike; $\bullet$, second additional spike; $\bullet$, third additional spike; $\square$, antidromic population spike in $\mathrm{Ca}^{2+}$-free medium. Responses in $\mathrm{Ca}^{2+}$-free medium were indistinguishable from those obtained in slices from control rats bathed in either medium. 


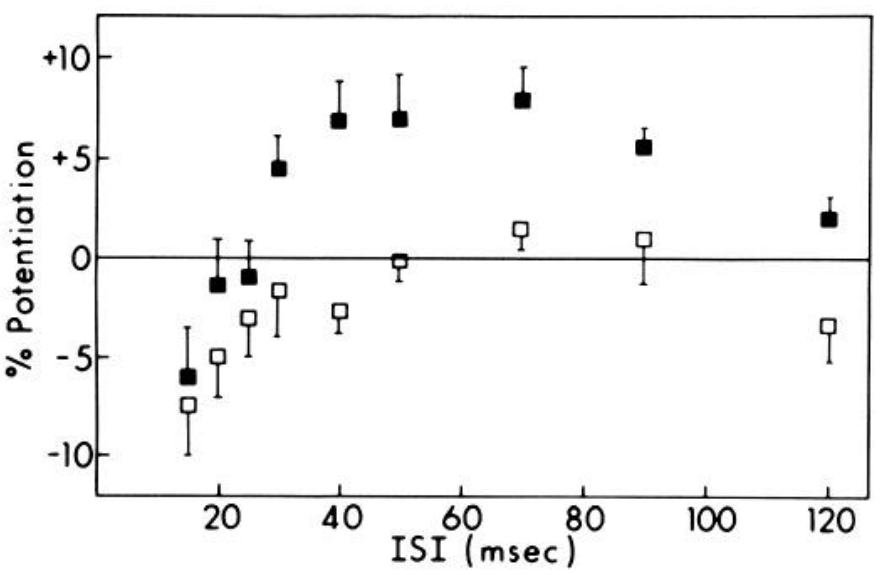

Figure 4. Responses of dentate granule cells to paired submaximal antidromic stimuli in slices from three KA-treated rats and the effect of $\mathrm{Ca}^{2+}$. free medium. These slices fired a single antidromic population spike. mean values in normal medium $( \pm \mathrm{SEM}) ; \square$, mean values in $\mathrm{Ca}^{2+}$-free medium. Two-way analysis of variance gave $p<0.01$ for effect of ISI and $p$ $<0.05$ for effect of $\mathrm{Ca}^{2+}$. Responses in $\mathrm{Ca}^{2+}$-free medium were indistinguishable from those obtained in slices from control rats bathed in either medium.

well as the underlying positive wave, but did not affect the initial (antidromic) spike (Fig. 3). The second and succeeding spikes exhibited paired-pulse potentiation, as did the underlying positive wave. Later spikes potentiated more than earlier spikes. A conditioning stimulus usually also increased the total number of spikes in the complex. Paired-pulse potentiation of the initial (antidromic) population spike was observed in these slices only when the antidromic spike was followed by just one additional population spike. When the first antidromic stimulus of the pair elicited several additional spikes, however, the amplitude of the second antidromic spike was less than that of the first, particularly with short ISIs (Fig. 3). Both effects of a conditioning stimulus on the antidromic population spike amplitude were $\mathrm{Ca}^{2+}$ dependent.

We attempted to reproduce the repetitive firing observed in slices from KA-treated rats by allowing control slices to deteriorate with time and by superfusing freshly prepared control slices with $100 \mu \mathrm{M}$ BMI. Multiple firing could be provoked by driving the mossy fibers antidromically $11.5 \mathrm{hr}$ after placing the slice in the chamber, just before all electrically evoked activity ceased. However, the second and succeeding population spikes were of low and variable amplitude and did not exhibit paired-pulse potentiation. Antidromic stimulation in the presence of BMl failed to elicit more than one population spike, however great the stimulus intensity. Thus, multiple firing of granule cell populations did not arise from deterioration of these neurons or simply from inactivation of GABAergic inhibition.

Slices from 10 additional KA-treated rats, like those from controls, generated only one antidromic population spike per stimulus, and paired-pulse depression was obtained with ISIs less than $30 \mathrm{msec}$ (Figs. 2 and 4). However, when pairs of antidromic stimuli with ISIs of 30 to $90 \mathrm{msec}$ were presented, the response to the second stimulus of the pair exceeded the response to the first by a maximum of 5 to $15 \%(p<0.01$ for effect of KA treatment compared to controls, $p<0.01$ for interaction between KA treatment and ISI, two-way analysis of variance). Superfusion of the slice with $\mathrm{Ca}^{2+}$. free medium reversibly eliminated paired-pulse potentiation of the antidromic spike (Fig. 4).

We frequently checked the response to antidromic stimulus pairs of other slices from the same animals with use of just one or two ISIs. All slices from the same rat gave the same result (none potentiated, all potentiated, or all generated multispike complexes).

\section{Histology}

Extent of $K A$ lesions. In a separate series of animals, we confirmed that neither intravenous nor ICV KA destroyed dentate granule cells.
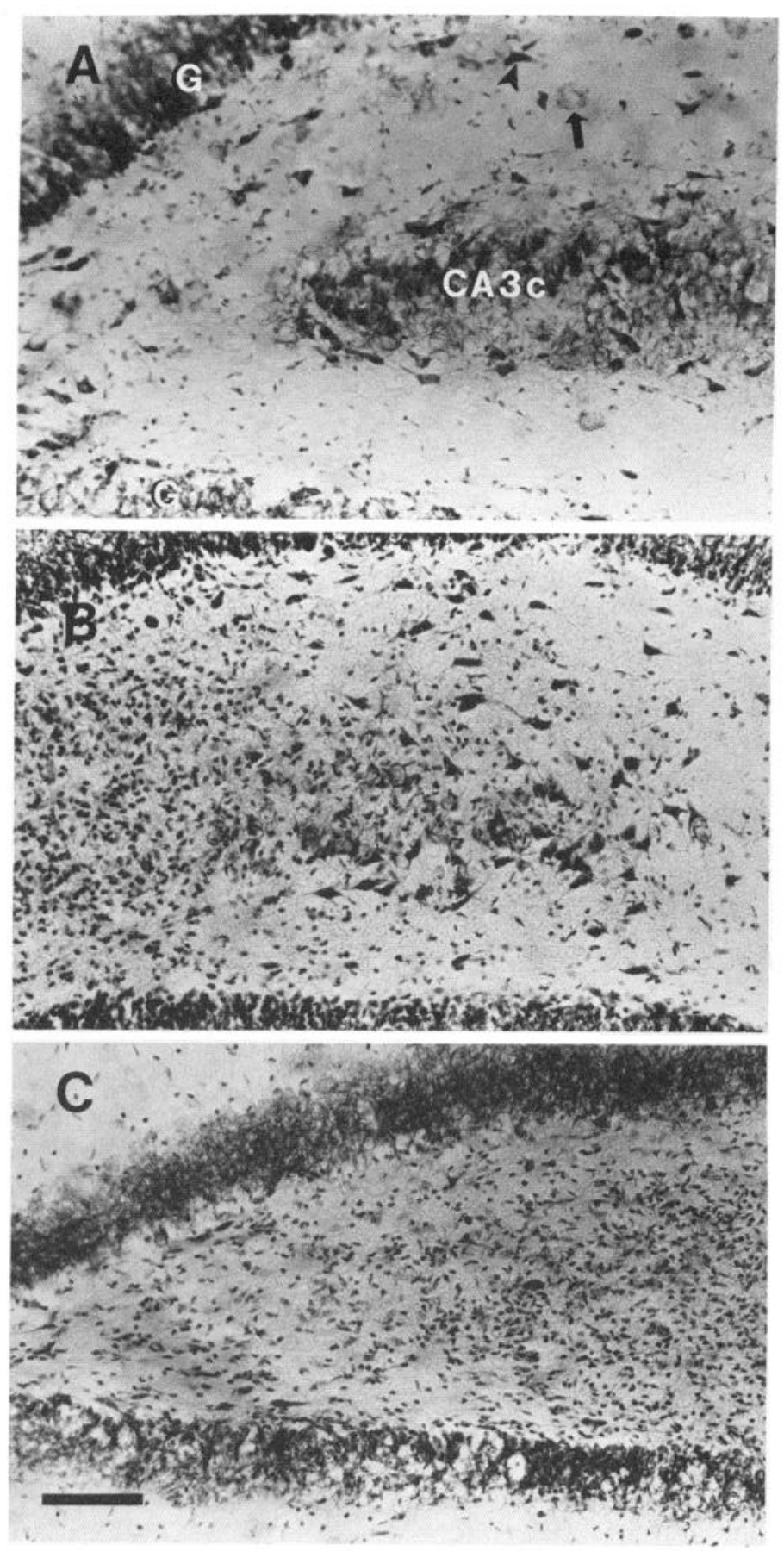

Figure 5. Loss of neurons from the dentate hilus 12 to 21 days after intravenous administration of $\mathrm{KA}(11 \mathrm{mg} / \mathrm{kg})$. A, Section from an animal injected with saline. The pyramidal cell layer of area CA3c and the granular layers $(G)$ are labeled. Area CA4 is the region between the granule and pyramidal cell layers. This region contains sparsely distributed normal-appearing (arrow) and dark (arrowhead) neurons. Dark cell transformation was considered artifactual and such neurons were counted as intact. $B$, Section from KA-treated rat that exhibited paired-pulse potentiation of the antidromic granule cell population spike, but fired no additional spikes. Neurons have been lost from the CA3-CA4 area, where there is substantial gliosis. $C$, Section from KA-treated rat that fired a multispike complex in response to antidromic stimulation of the mossy fibers. Neurons are virtually absent from areas CA4 and CA3c. These regions are gliotic and the dentate hilus has shrunken. Cresyl violet stain. Calibration: $0.1 \mathrm{~mm}$. 


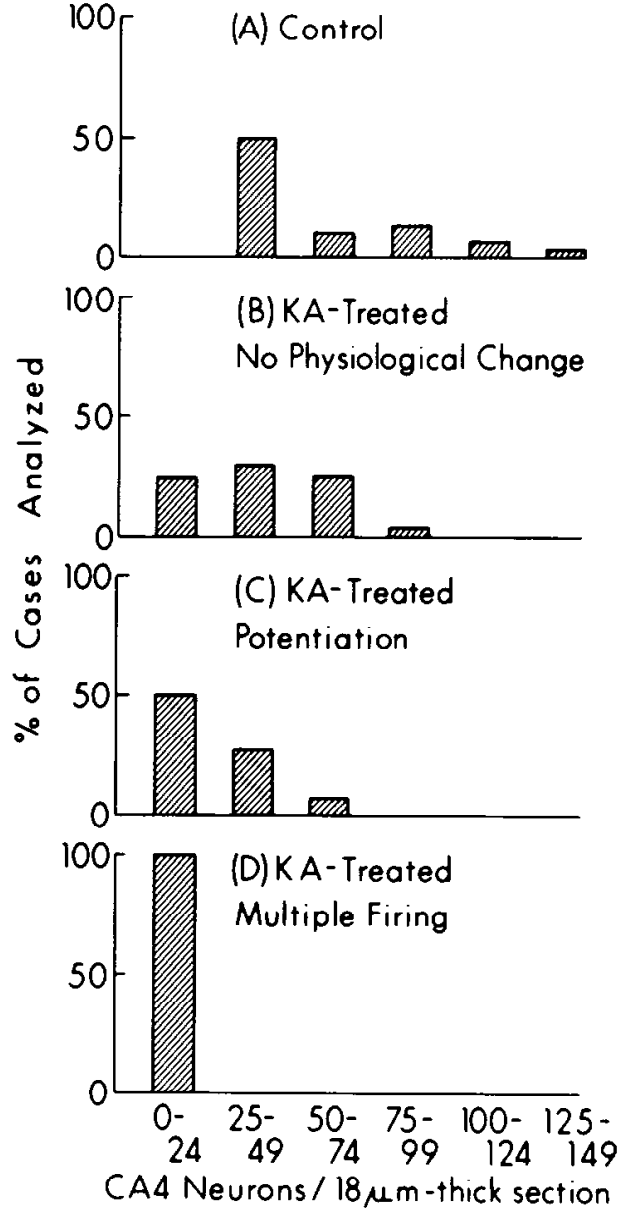

Figure 6. Number of CA4 neurons in sections cut from temporal hippocampal slices 12 to 21 days after KA administration. Data from KA-treated rats are related to their electrophysiological response to paired antidromic stimulation of the mossy fibers. Number of animals: $A, 19 ; B, 16 ; C, 10 ; D$, 5. Group $B$ differed from controls at $p<0.05$, and groups $C$ and $D$ differed from controls at $p<0.01$ (one-way analysis of variance).

Of the neurons that project to the fascia dentata, only those in area CA4 degenerated in any significant number.

Our cell counts clearly demonstrated that KA administration reduced the number of CA4 neurons in a manner that correlated with the electrophysiological data (Figs. 5 and 6). Sections from animals that fired multiple population spikes in response to single antidromic stimuli contained fewer CA4 neurons than any of the sections from control animals. Sections from the other two groups of KA-treated rats contained more CA4 neurons, but still significantly fewer than controls.

Growth of supragranular mossy fiber collaterals. Sparsely distributed supragranular mossy fiber collaterals were often, but not always, observed at the location chosen for electrophysiological recordings in sections cut from control slices (Figs. $1 A$ and 7). In contrast, a moderate-to-dense band of these fibers was always present in sections from animals that fired multiple population spikes in response to a single antidromic stimulus (Figs. $1, C$ and $D$, and 7). Individual mossy fibers could frequently be followed from their origin beneath the granular layer through this layer to the supragranular zone. Similarly extensive mossy fiber growth was evident in half the cases that exhibited paired-pulse potentiation but fired just a single population spike. Of $13 \mathrm{KA}$-treated rats that responded to antidromic stimulation of the mossy fibers indistinguishably from controls, only 3 showed unequivocal evidence of mossy fiber collateral growth.

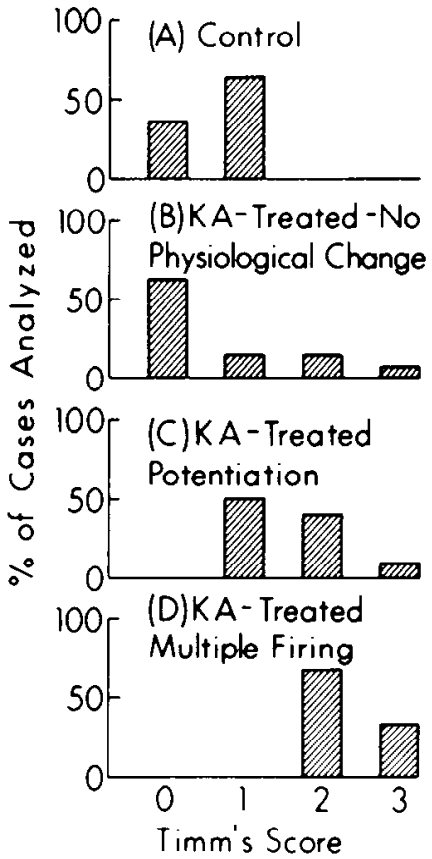

Figure 7. Timm's scores of sections cut from hippocampal slices 12 to 21 days after KA administration. See "Materials and Methods" and Figure 1 for scoring criteria. Data from KA-treated rats are related to their electrophysiological response to paired antidromic stimulation of the mossy fibers. Number of animals: $A, 22 ; B, 13 ; C, 10 ; D, 6$. Groups $B, C$, and $D$ differed from controls at $p<0.01$ (Fisher's exact test).

\section{Discussion}

Our results demonstrate that intravenous or ICV administration of KA can alter the response of dentate granule cells to stimulation of their axons. Normally, the granule cells fire a single antidromic population spike whose amplitude is not increased by an antidromic conditioning stimulus. Two changes were recorded in the present study. First, granule cells in slices from some KA-treated rats fired multiple population spikes in response to a single antidromic stimulus. Second, in slices from many KA-treated rats, a conditioning antidromic stimulus potentiated the response to an identical test stimulus. All effects of KA administration were $\mathrm{Ca}^{2+}$ dependent.

The most persuasive evidence that recurrent mossy fiber collaterals mediated these abnormal granule cell responses is the excellent correlation between electrophysiological and histological data. In general, the greater the loss of CA4 neurons, the more dense was the growth of supragranular mossy fiber collaterals and the more abnormal was the response to antidromic mossy fiber stimulation. Only slices from animals with clear evidence of recurrent mossy fiber collateral growth were capable of firing multiple population spikes and slices from five other such animals exhibited pairedpulse potentiation of single antidromic spikes. In no case did a slice that lacked supragranular mossy fiber collaterals exhibit these responses. The lack of abnormal electrophysiological response in slices from three rats which nevertheless had developed extensive recurrent collaterals may perhaps be explained by excessive damage to the relatively fragile giant mossy fiber boutons in cutting those particular slices. In addition, the action of nascent mossy fiber synapses may sometimes have been masked by other, as yet unknown, consequences of KA administration.

Other lines of evidence also tend to support synaptic mediation of these KA-induced electrophysiological changes. Both paired-pulse potentiation and repetitive firing were reversibly abolished by superfusion with $\mathrm{Ca}^{2+}$-free medium. Although dependence of an evoked response on extracellular $\mathrm{Ca}^{2+}$ does not in itself prove the involvement of a synaptic mechanism, it remains nonetheless one of the essential criteria. In addition, antidromically evoked multispike complexes evidenced substantial paired-pulse potentiation. This form of 
synaptic plasticity characterizes excitatory transmission in many hippocampal pathways, particularly in the mossy fiber pathway (Yamamoto et al., 1980). The maximum duration of paired-pulse potentiation observed in the present study (at least $100 \mathrm{msec}$ ) was well within the range reported by Yamamoto et al. (1980) at mossy fiber synapses in area CA3.

We suggest that, in slices from KA-treated rats, the first antidromic stimulus of the pair fired some granule cells by initiating action potentials in their mossy fibers and depolarized some by activating recurrent mossy fiber projections onto the apical dendrite. When a high density of recurrent synapses had been formed, the synaptic depolarization could have been sufficient to generate a multispike complex through repetitive activation of the recurrent pathway. In many of these same slices we also observed $\mathrm{Ca}^{2+}$-dependent paired-pulse depression of the antidromic population spike, even as the following, presumably orthodromic, spikes exhibited potentiation. The second antidromic population spike may have been attenuated in these cases by prolonged depolarization and/or conductance shunting associated with the first recurrent EPSP. Finally, $\mathrm{Ca}^{2+}$. dependent paired-pulse potentiation of single antidromically evoked population spikes appeared to be associated with the formation of fewer recurrent mossy fiber synapses than were needed to support multiple firing. Our results do not, however, indicate a clear mechanism by which recurrent synaptic excitation could have produced this enhancement. A final resolution of these mechanistic issues awaits intracellular recording of the recurrent EPSP and the discovery of a selective blocker of mossy fiber transmission.

Two factors other than reinnervation might be thought to have contributed to the increased antidromically evoked firing we observed. One is a loss of recurrent inhibition. Stimulation of mossy fibers provokes feedback inhibition onto granule cell bodies via synaptic activation of the GABAergic basket cells (Andersen et al., 1966; Storm-Mathisen, 1977). We have found little histological evidence of basket cell degeneration after intravenous or ICV administration of KA. However, some of the deeper CA4 neurons that are destroyed by KA administration appear to be GABAergic and probably contribute an inhibitory component to the associational-commissural projection (Seress and Ribak, 1983). Other groups have observed a depression of synaptic IPSPs in hippocampal pyramidal cells 2 weeks after ICV administration of KA (Franck and Schwartzkroin, 1983; Lancaster et al., 1983). However, our failure to duplicate the consequences of KA administration by superfusing control slices with BMl (see also Fricke and Prince, 1984) indicates that the loss of recurrent inhibition could not in itself have accounted for antidromically evoked multiple spike firing. Additionally, recurrent inhibition appeared not to be involved in paired-pulse interactions when both stimuli of the pair were antidromic. Evidently, the conductance increase associated with the recurrent granule cell IPSP could not block the somatic invasion of the antidromic action potential or appreciably reduce its amplitude. Therefore, the $\mathrm{Ca}^{2+}$-insensitive paired-pulse depression obtained with short ISIs in all slices from both control and KA-treated rats probably reflected intrinsic properties of the granule cell membrane. Assaf et al. (1981) reported a similar paired-pulse depression of antidromic action potential amplitude in single granule cells when the ISI was less than $30 \mathrm{msec}$, that is, when the second action potential reached the soma during the depolarizing afterpotential. On the basis of this evidence, the pairedpulse depression obtained in the present study may have reflected the attenuation of granule cell action potentials by a combination of the membrane depolarization and marked decrease in membrane resistance associated with the afterpotential. $\mathrm{Ca}^{2+}$-sensitive pairedpulse potentiation did not replace the depression, but appeared to be superimposed upon it (Figs. 3 and 4 ).

Another possible factor that must be considered is a KA-induced change in the biophysical properties of the granule cell membrane. Conceivably, any of several such changes could have contributed to the increased excitability we observed, including enhancement of the depolarizing afterpotential (Assaf et al., 1981), suppression of a voltage-sensitive outward current (Fricke and Prince, 1984), or enhancement of electrotonic coupling among granule cells (MacVicar and Dudek, 1982). Our data neither support nor eliminate these possibilities. Intracellular recordings are obviously required for this purpose.

Synaptic rearrangements almost inevitably follow brain damage in experimental animals (Cotman et al., 1981). There is some controversy as to whether the nascent connections primarily serve to reinstitute normal physiological function in the damaged brain region or to mediate abnormal function. Our results appear to support the view that lesion-induced synaptogenesis can create circuitry that is physiologically maladaptive. Recurrent mossy fiber collaterals, if sufficient in number, can evidently mediate reverberating excitation within the fascia dentata. It seems likely that such abnormal excitability would further compromise hippocampal function that had already been disrupted by neuronal degeneration. For example, in light of evidence that the fascia dentata normally dampens high frequency discharges in the limbic circuit (Collins et al., 1983), one would predict that the formation of recurrent mossy fibers would lead to a reduction in seizure threshold. The development of aberrant recurrent excitatory circuits might thus account, in part, for the spontaneous behavioral and/or electrographic seizures that we and others (Pisa et al., 1980; Cavalheiro et al., 1982) have observed weeks after administering a lesioning dose of KA. In this regard, a recent report of aberrant supragranular mossy fibers in aged human hippocampi (Cassell and Brown, 1984) is especially provocative.

\section{References}

Andersen, P., B. Holmqvist, and P. E. Voorhoeve (1966) Entorhinal activation of dentate granule cells. Acta Physiol. Scand. 66: 448-460.

Andersen, P., T. V. P. Bliss, and K. K. Skrede (1971) Unit analysis of hippocampal population spikes. Exp. Brain Res. 13: 208-221.

Assaf, S. Y., V. Crunelli, and J. S. Kelly (1981) Electrophysiology of the rat dentate gyrus in vitro. In Electrophysiology of /solated Mammalian CNS Preparations, G. A. Kerkut and H. V. Wheal, eds., pp. 153-187, Academic Press, Inc., New York.

Ault, B., and J. V. Nadler (1983) Anticonvulsant-like actions of baclofen in the rat hippocampal slice. Br. J. Pharmacol. 78: 701-708.

Berger, T. W., S. Semple-Rowland, and J. L. Bassett (1981) Hippocampal polymorph neurons are the cells of origin for ipsilateral association and commissural afferents to the dentate gyrus. Brain Res. 215: 329-336.

Cassell, M. D., and M. W. Brown (1984) The distribution of Timm's stain in the non-sulphide-perfused human hippocampal formation. J. Comp. Neurol. 222: 461-471

Cavalheiro, E. A., 'D. A. Riche, and G. Le Gal La Salle (1982) Long-term effects of intrahippocampal kainic acid injection in rats: A method for inducing spontaneous recurrent seizures. Electroencephalogr. Clin. Neurophysiol. 53: 581-589.

Collins, R. C., R. G. Tearse, and E. W. Lothman (1983) Functional anatomy of limbic seizures: Focal discharges from medial entorhinal cortex in rat. Brain Res. 280: 25-40.

Corsellis, J. A. N., and B. S. Meldrum (1976) Epilepsy. In Greenfield's Neuropathology, W. Blackwood and J. A. N. Corsellis, eds., pp. 771-795, Edward Arnold, London.

Cotman, C. W. and J. V. Nadler (1978) Reactive synaptogenesis in the hippocampus. In Neuronal Plasticity, C. W. Cotman, ed., pp. 227-271, Raven Press, New York.

Cotman, C. W., M. Nieto-Sampedro, and E. W. Harris (1981) Synapse replacement in the nervous system of adult vertebrates. Physiol. Rev. 61: 684-784.

Danscher, G. (1981) Histochemical demonstration of heavy metals. A revised version of the sulphide silver method suitable for both light and electronmicroscopy. Histochemistry 71: 1-16.

De Lorenzo, R. J., and G. H. Glaser (1981) Neuropathologic changes and neuronal plasticity in temporal lobe-limbic epilepsy. Neurology 31:114.

De Lorenzo, R. J., G. H. Glaser, P. DeLucia, and D. Schwartz (1982) The role of neuronal plasticity in epilepsy. Neurology 32: A.92

Elliott, K. A. C. (1969) The use of brain slices. In Handbook of Neurochemistry, A. Lajtha, ed., Vol. 2, pp. 103-114, Plenum Press, New York.

Franck, J. E., and P. A. Schwartzkroin (1983) Kainate lesioned hippocampi become epileptogenic. Soc. Neurosci. Abstr. 9: 908. 
Fricke, R. A., and D. A. Prince (1984) Electrophysiology of dentate gyrus granule cells. J. Neurophysiol. 51: 195-209.

Frotscher, M., and J. Zimmer (1983) Lesion-induced mossy fibers to the molecular layer of the rat fascia dentata: Identification of postsynaptic granule cells by the Golgi-EM technique. J. Comp. Neurol. 215: 299-311.

Gaarskjaer, F. B., and S. Laurberg (1983) Ectopic granule cells of hilus fasciae dentatae projecting to the ipsilateral regio inferior of the rat hippocampus. Brain Res. 274: 11-16.

Gottlieb, D. I., and W. M. Cowan (1973) Autoradiographic studies of the commissural and ipsilateral association connections of the hippocampus and dentate gyrus of the rat. I. The commissural connections. J. Comp. Neurol. 149: 393-422.

Haug, F. - M. S. (1973) Hcavy metals in the brain. A light microscope study of the rat with Timm's sulphide silver method. Methodological considerations and cytological and regional staining patterns. Adv. Anat. Embryol. Cell Biol. 47: 1-71.

Lancaster, B., H. V. Wheal, and T. J. Ashwood (1983) Hippocampal electrophysiology after kainic acid treatment: A chronic model of focal epilepsy. Soc. Neurosci. Abstr. 9: 908.

Laurberg, S., and K. E. Sørensen (1981) Associational and commissural collaterals of neurons in the hippocampal formation (hilus fasciae dentatae and subfield CA3). Brain. Res. 212: 287-300.

Laurberg, S., and J. Zimmer (1981) Lesion-induced sprouting of hippocampal mossy fiber collaterals to the fascia dentata in developing and adult rats. J. Comp. Neurol. 200: 433-459.

MacVicar, B. A., and F. E. Dudek (1982) Electrotonic coupling between granule cells of rat dentate gyrus: Physiological and anatomical evidence. J. Neurophysiol. 47: 579-592.

Nadler, J. V. (1981) Kainic acid as a tool for the study of temporal lobe epilepsy. Life Sci. 29: 2031-2042.

Nadler, J. V., B. W. Perry, and C. W. Cotman (1980) Selective reinnervation of hippocampal area CA1 and the fascia dentata after destruction of CA3 CA4 afferents with kainic acid. Brain Res. 182: 1-9.

Nadler, J. V., D. L. Tauck, D. A. Evenson, and J. N. Davis (1983) Synaptic rearrangements in the kainic acid model of Ammon's horn sclerosis. In Excitotoxins, Wenner-Gren International Symposium 39, K. Fuxe, P. J. Roberts, and R. Schwarcz, eds., pp. 256-270, MacMillan Press, Houndmills.

Pisa, M., P. R. Sanberg, M. E. Corcoran, and H. C. Fibiger (1980) Spontaneous recurrent seizures after intracerebral injections of kainic acid in rats: A possible model of human temporal lohe epilepsy. Brain Res. 200: 481 487.

Seress, L., and C. E. Ribak (1983) GABAergic cells in the dentate gyrus appear to be local circuit and projection neurons. Exp. Brain Res. 50: 173182.

Storm-Mathisen, J. (1977) Localization of transmitter candidates in the brain: The hippocampal formation as a model. Prog. Neurobiol. 8: 119-181.

Swanson, L. W., J. M. Wyss, and W. M. Cowan (1978) An autoradiographic study of the organization of intrahippocampal association pathways in the rat. J. Comp. Neurol. 181: 681-716.

Tauck, D. L. (1983) Aberrant recurrent excitatory circuit in the fascia dentata of rats following kainic acid lesions. Ph.D. thesis, Duke University, Durham, NC.

Tauck, D. L., and J. V. Nadler (1982) Electrophysiological evidence that hippocampal mossy fibers form an aberrant recurrent excitatory circuit after kainic acid lesion. Soc. Neurosci. Abstr. 8: 744.

White, W. F., J. V. Nadler, and C. W. Cotman (1978) A perfusion chamber for the study of CNS physiology and pharmacology in vitro. Brain Res. 152: 591-596.

Yamamoto, C., K. Matsumoto, and M. Takagi (1980) Potentiation of excitatory postsynaptic potentials during and after repetitive stimulation in thin hippocampal sections. Exp. Brain Res. 38: 469-477. 